Revista de Psicología Vol. 31 (2), 2013 (ISSN 0254-9247)

\title{
El ajuste instructivo entre estilos de aprendizaje y enseñanza en la universidad
}

\author{
Ana Clara Ventura ${ }^{1}$ \\ Instituto Rosario de Investigaciones en Ciencias de la Educación, \\ Consejo Nacional de Investigaciones Cientificas y Técnicas
}

\begin{abstract}
Se presenta una revisión de artículos empíricos sobre el ajuste instructivo entre estilos de aprendizaje y estilos de enseñanza en la universidad (2002-2012). Se consultaron las bases de datos Dialnet, Redalyc, Scielo y Doaj debido a su impacto en el contexto latinoamericano. Los descriptores fueron: "estilos de aprendizaje", "estilos de enseñanza" y "universidad". Se identificaron 53 artículos, de los cuales 17 cumplían los criterios de inclusión (artículo: empírico; muestra: universitaria, objeto: ajuste estilos de aprendizaje y de enseńanza, recorte temporal, idioma espańol). La revisión de la literatura mostró que el ajuste se investiga, entiende y aborda desde dos líneas: (1) la instrucción adaptativa o (2) la estilística. Se discuten los resultados.

Palabras clave: instrucción adaptativa, enfoque estilístico, educación superior
\end{abstract}

\section{The hypothesis of matching about styles of learning and teaching at Higher Education} A review of learning and teaching styles matching at university over the last ten years is presented. For this purpose, a review was carried out of the Dialnet, Redalyc, Scielo and Doaj data bases due to their scope and importance in the Hispanic context. The descriptors used in the search for information were the key words: learning styles, teaching styles and higher education. The data bases consulted produced a total of 53 papers, with only 17 of these complying with all the criteria for inclusion (paper: empirical research; sample: university; object: correspondence learning and teaching styles; time period 2002-2012; Spanish language). The literature review showed that the matching is researched and understanding from two approaches: (1) adaptive instruction or (2) stylistics. Results are discussed. Key words: adaptative instruction, stylistic approach, higher education

1 Licenciada y profesora universitaria en Psicopedagogía. Becaria doctoral del Consejo Nacional de Investigaciones Científicas y Técnicas (CONICET, Argentina), cumple funciones en IRICE. Dirección: 27 de febrero 210 bis. CP: 2000, Rosario, Santa Fe, Argentina. Correo electrónico: ventura@irice-conicet.gov.ar 

En la última década, diversas investigaciones psicoeducativas demostraron que los estudiantes tienden a encaminarse hacia un estilo particular de aprendizaje que va de acuerdo a sus características y habilidades individuales (Blumen, Rivero \& Guerrero, 2011; Evans, Cools \& Charlesworth, 2010; González Lomelí, Castañeda Figueiras \& Maytorena Noriega, 2000, Komarraju, Karau, Schmeck \& Avdic, 2011; Peterson, Rayner \& Armstrong, 2009). De acuerdo con ello, prefieren ciertas estrategias de enseñanza de sus docentes (Bertoglia Richards, 2005; Gravini Donado, Cabrera Pérez, Avila Molina \& Vargas González, 2009). En el ámbito universitario, "actualmente, al igual que hace ya décadas ocurrió para niveles educativos inferiores, están proliferando investigaciones que examinan las influencias recíprocas de las acciones del profesor y del estudiante" (Prados Gallardo, Cubero Pérez \& de la Mata Benítez, 2010, p. 167). En este marco, durante las últimas dos décadas del siglo XX (1980-1999), la problemática en torno a la interacción entre estilos de aprendizaje y estilos de enseñanza versó sobre la discusión de ajuste o no ajuste instructivo. $\mathrm{El}$ ajuste instructivo se define como el proceso interactivo que resulta del emparejamiento entre el estilo de enseńanza del profesor y el estilo de aprendizaje del estudiante. Es una variable proceso que incidiría en los logros de aprendizaje del alumnado (Serrano Pastor, 1994). Actualmente, ha recibido la denominación de tendencias armonizadoras o discrepantes entre aprendizaje y enseñanza (Salvador, Argos González, Ezquerra Muñoz, Osoro Sierra \& Castro, 2011).

En la última década, el problema de ajuste-no ajuste no parecería ubicarse en el núcleo del debate dado que la mayor parte de los trabajos de investigación parten del supuesto de que la correspondencia entre estilos de aprendizaje y estilos de enseñanza es beneficiosa para promover la alfabetización académica (Esguerra Pérez \& Guerrero Ospina, 2010; Hervás Aviles, 2003; Joyce, Weil \& Calhoun, 2000; 
Martínez Geijo, 2008; Ruiz Ruiz, Trillos Gamboa \& Morales Arrieta, 2006). En otros términos, es posible observar que el foco de la problemática giró hacia los modos de entender y/o abordar el ajuste entre estilos de aprendizaje y estilos de enseñanza. Según Clariana (2001), se pueden distinguir al menos dos modalidades:

1. La correspondencia entre los métodos de enseñanza, las actividades o los materiales elaborados por el docente y las preferencias de estilo de los estudiantes, esto significaría básicamente el ajuste del estilo docente a sus alumnos.

2. La correspondencia entre las mismas preferencias o estilos entre docentes y estudiantes.

Es posible interpretar que en la primera alternativa de correspondencia se produce la adaptación del estilo pedagógico del docente al estilo de sus estudiantes, mientras que en la segunda modalidad prevalecería la orientación inversa, la modificación de los estilos de aprendizaje de los estudiantes (Hederich Martínez, Gravini Donado \& Camargo Uribe, 2011). En esta línea de pensamiento, el objeto de esta revisión centra la atención sobre los estudios empíricos que analizan la interacción entre estilos de aprendizaje y estilos de enseńanza en entornos universitarios. Para ello, se realizó un análisis descriptivo de documentos reportados en la última década (del año 2002 al 2012) en distintas bases de datos con un relativo alcance e importancia en el contexto académico e investigativo latinoamericano: Dialnet, Redalyc, Scielo y Doaj. Los descriptores utilizados fueron estilos de aprendizaje, estilos de enseñanza (o en su defecto: "preferencias instruccionales", "enseñanza”; “educación”) y universidad. Esta búsqueda identificó 53 artículos de los cuales 36 fueron excluidos por no cumplir con los siguientes criterios: objeto de e estudio (interacción estilos de aprendizaje y enseñanza), tipo de artículo (investigación empírica), tipo de población/muestra (universitaria) y recorte temporal (2002-2012). 


\section{Tabla 1}

Articulos excluidos: caracteristicas y distribución

\begin{tabular}{llc}
\hline Criterios & Artículos excluidos & Total \\
\hline Objeto de estudio & Estilos de aprendizaje & 17 \\
Tipo de artículo & Reflexión, revisión o propuesta metodológica & 14 \\
$\begin{array}{l}\text { Tipo de población/ } \\
\text { muestra }\end{array}$ & Nivel educativo primario/ secundario & 4 \\
Recorte temporal & Ańo 2000 & 1 \\
\hline
\end{tabular}

De aquí que se analizaron un total de 17 trabajos empíricos dirigidos a estudiar la interacción entre los estilos de aprendizaje y enseñanza en el ámbito universitario que han sido reportados durante la última década (2002-2012). La distribución de artículos recuperados según las bases consultadas ha sido: Dialnet (12), Scielo (4), Redalyc (1), Doaj (0). El análisis del corpus se analizó y clasificó en sucesivas fases. En primer lugar, se describieron las características generales de los artículos. En esta primera aproximación, se hallaron básicamente dos líneas de investigación: (1) el análisis de los perfiles de estilos de aprendizaje y de enseñanza desde una perspectiva unidireccional (estudiantes o docente) y (2) el análisis de la interacción áulica propiamente dicha desde una perspectiva bidireccional (estudiantes y docentes). Estas líneas fueron denominadas como Tipo I y Tipo II, respectivamente. En este sentido, el reporte de los resultados se organizó de acuerdo a su perspectiva o tipo de investigación. En segundo lugar, se analizó el modo en que las investigaciones proponen abordar y responder a la problemática de la correspondencia entre estilos de aprendizaje y enseñanza. Por último, se identificaron las futuras propuestas de investigación e implicancias educativas que aparecen detalladas explícitamente en los trabajos de investigación. 


\section{Descripciones generales del corpus}

A nivel teórico, las investigaciones refieren a distintos constructos que forman parte de un mismo objeto de estudio: un conjunto de rasgos propios que determinan formas o características preferidas de aprender y enseñar relativamente estables. Las variables halladas son: estilos de aprendizaje (Es-A), enfoques de aprendizaje (En-A), estilos de docencia (Es-D), estilos de enseñanza (Es-E), preferencias de enseñanza (Pr-E), metodologías de enseñanza (ME), métodos instruccionales (MI), estrategias de enseñanza (Est-E), satisfacción de actividades didácticas (SAD) y guías didácticas docentes (GDD). A nivel metodológico, predominan los estudios descriptivos-correlacionales de corte transversal con aplicación de instrumentos de autoinforme (cuestionarios, inventarios, escalas) preexistentes o construidas ad hoc. Entre los instrumentos estandarizados, se empleó recurrentemente el Cuestionario Honey-Alonso de Estilos de Aprendizaje (CHAEA) apoyando los resultados de revisiones actuales sobre su uso (Bahamón Muñetón, Vianchá Pinzón, Alarcón Alarcón \& Bohórquez Olaya, 2012). Asimismo, aunque con menor frecuencia, se hallaron estudios cualitativos que emplearon técnicas como observación y entrevista.

\section{Análisis unidireccional sobre los estilos de aprendizaje y enseńanza}

Se hallaron diez artículos publicados sobre la investigación de estilos de aprendizaje y enseñanza desde una perspectiva unidireccional (véase Tabla 2). Esto es, el problema se focalizó sobre uno de los actores implicados en el proceso: el aprendizaje y la enseńanza desde la perspectiva de los estudiantes o de los docentes. En este sentido, el núcleo de los artículos versa fundamentalmente sobre el análisis de procesos y estilos de aprendizaje de estudiantes de diferentes disciplinas (educación, psicología, medicina, física, historia, geografía) así como sobre sus preferencias instruccionales. El período temporal de las investigaciones 
de esta clase se encuentra entre 2004-2011 y la mayor parte de ellas se radicaron en el contexto universitario español. Cabe señalar que desde el año 2009 las publicaciones acerca de esta temática se incrementaron notablemente.

\section{Tabla 2}

Caracteristicas de los artículos tipo I (unidireccionales) según año de publicación

\begin{tabular}{|c|c|c|}
\hline Año & $\begin{array}{l}\text { Contexto } \\
\text { universitario* }\end{array}$ & Tamaño y tipo de muestra \\
\hline 2004 & México & 105 estudiantes de lenguas extranjeras \\
\hline 2007 & España & 136 estudiantes de educación \\
\hline 2008 & España & 158 estudiantes de magisterio \\
\hline 2009 & España & 111 estudiantes de psicología \\
\hline 2009 & Chile & 72 estudiantes de medicina \\
\hline 2009 & España & 166 docentes de diferentes titulaciones \\
\hline 2010 & México & 30 docentes de física \\
\hline 2010 & México & $\begin{array}{l}\text { Docentes de Ingeniería. No se informó tamaño de } \\
\text { muestra }\end{array}$ \\
\hline 2011 & España & $\begin{array}{l}278 \text { estudiantes de educación, historia, geografía } \\
\text { y medicina }\end{array}$ \\
\hline 2011 & España & 161 estudiantes de pedagogía \\
\hline
\end{tabular}

Nota: * Filiación del primer autor del artículo

Tal como puede apreciarse en la Tabla 3, se presentan los referentes de cada artículo junto con una breve descripción de las investigaciones unidireccionales o tipo I (variables, instrumentos y resultados principales). 


\section{Tabla 3}

Revisión sobre las investigaciones Tipo I

\begin{tabular}{|c|c|c|}
\hline Autores & $\begin{array}{l}\text { Variables e } \\
\text { instrumentos }\end{array}$ & Resultados principales \\
\hline $\begin{array}{l}\text { Hernández } \\
\text { Ruiz } \\
(2004)\end{array}$ & $\begin{array}{l}\text { Es-A } \\
\text { (Cuestionario } \\
\text { sobre Estilo de } \\
\text { Aprendizaje } \\
\text { Perceptivo y } \\
\text { Predominio } \\
\text { Hemisférico- } \\
\text { Cerebral) }\end{array}$ & $\begin{array}{l}\text { Los estudiantes entre } 17 \text { y } 32 \text { años de edad de las áreas } \\
\text { económico-administrativas, matemáticas, humanida- } \\
\text { des y ciencias químico-biológicas, mostraron un mayor } \\
\text { predominio del estilo cinestético. Los estudiantes entre } \\
41 \text { y } 48 \text { años de edad y los que estudian un doctorado } \\
\text { mostraron mayor predominio por el estilo grupal. Los } \\
\text { estudiantes de maestría y de ciencias sociales mostraron } \\
\text { mayor predominio del estilo táctil. Los estudiantes entre } \\
33 \text { y } 40 \text { ańos de edad mostraron mayor predominio del } \\
\text { estilo visual. }\end{array}$ \\
\hline $\begin{array}{l}\text { Berbén } \\
\text { et al. } \\
(2007)\end{array}$ & $\begin{array}{l}\text { En-A } \\
\text { (R-SPQ-2F). } \\
\text { Pr-E (USET) }\end{array}$ & $\begin{array}{l}\text { El alumnado que prefiere clases magistrales adopta una } \\
\text { posición de enfoque más superficial. En cambio, los } \\
\text { participantes que principalmente: desean las drama- } \\
\text { tizaciones como método de enseñanza y esperan y/o } \\
\text { rechazan una enseńanza basada en el trabajo en grupo, } \\
\text { se caracterizaron por un aprendizaje más profundo. }\end{array}$ \\
\hline
\end{tabular}

Se hallaron asociaciones significativas entre las preferencias por métodos bidireccionales (centrados en el aprendiz y con altos grados de interacción entre los Es-A aprendices, incluye "grupos de discusión", "simulacio(Inventory of nes", "role-playing", "aprendizaje cooperativo" y "juegos

García Learning

Ros et al. Processes)

(2008)

MI

(Escala ad hoc) instruccionales") y unidireccionales (centrados en el profesor, caracterizados por la interacción en el sentido profesor-alumno, incluye los métodos de "tutorización individual", "resolución de problemas", "demostraciones" y "práctica y ejercitación") y el procesamiento elaborativo, así como una relación inversa entre preferencias por métodos bidireccionales y retención de hechos.

$\begin{array}{ll}\text { Pulido } & \text { Es-A/Es-E } \\ \text { Martos et } & \text { (Cuestionario } \\ \text { al. (2009) } & \text { ad hoc) }\end{array}$

Los estudiantes prefirieron las estrategias relacionadas con los estilos pragmático y activo.

$\begin{array}{ll}\text { Martos et } & \text { (Cuestion } \\ \text { al. (2009) ad hoc) }\end{array}$ 


\begin{tabular}{|c|c|c|}
\hline Autores & $\begin{array}{l}\text { Variables e } \\
\text { instrumentos }\end{array}$ & Resultados principales \\
\hline $\begin{array}{l}\text { Villalobos- } \\
\text { Alarcón } \\
\text { et al. } \\
(2009)\end{array}$ & $\begin{array}{l}\text { Es-A (CHAEA). } \\
\text { ME } \\
\text { (Encuesta de } \\
\text { satisfacción de } \\
\text { metodologías de } \\
\text { enseńanza) }\end{array}$ & $\begin{array}{l}\text { El perfil predominante de los estudiantes fue el reflexivo } \\
\text { y, en segundo lugar, el teórico. Las metodologías de } \\
\text { enseñanza preferidas fueron el uso de modelos y la clase } \\
\text { expositiva. Los EA no se hallaron correlacionados a las } \\
\text { preferencias instruccionales de enseñanza. }\end{array}$ \\
\hline
\end{tabular}

Martínez

Geijo

(2009)
Es-E

(Escala $a d$ hoc)

El estilo de enseñanza formal y funcional de los docentes, favorecen el estilo de aprendizaje reflexivo y pragmático de los estudiantes.

El estilo de enseńanza y aprendizaje preferente de los docentes parece estar influido por la titulación.

Los profesores desconocían la existencia de estilos de aprendizaje y tenían ciertos preconceptos equivocados. El sistema 4MAT de enseñanza basado en los estilos de aprendizaje de los estudiantes fue una gran herramienta para la enseñanza de la física, sin embargo, no estuvo

Ramírez

Díaz y Es-A/Es-E

Chávez (Cuestionario

Lima ad hoc)

(2010) exento de dificultades en su aplicación: 1. El tiempo requerido fue superior al destinado por el programa de la materia. 2. Ausencia de recursos materiales y físicos. 3. La renuencia de algunos profesores de adoptar este tipo de estrategias, señalando que es una especie de "moda" derivada de la "imposición" del modelo educativo institucional. 4. La falta de pericia de los profesores en el manejo de los estilos de aprendizaje, que al ser la primera vez que lo implementaban solían tener dificultades y caían fácilmente en regresar a su práctica tradicional "sin notarlo".

El estilo predominante de enseñanza fue el formal o tipo II. Se caracteriza por el dictado de clases teóricas tradi-

Ramírez

Díaz y Es-E

Chávez (Cuestionario

Lima ad hoc)

(2010) cionales, transmitir la sabiduría, ser exactos, presentar la información sistemáticamente, basarse en hechos y detalles, así como en un tipo de pensamiento secuencial y organizacional, el uso tradicional de la autoridad. Los estudiantes que se sintieron más cómodos fueron aquellos que son disciplinados, su atención se centra en el material, cuando se presentan los ejemplos son los primeros en presentar dudas. 


\begin{tabular}{|c|c|c|}
\hline Autores & $\begin{array}{l}\text { Variables e } \\
\text { instrumentos }\end{array}$ & Resultados principales \\
\hline $\begin{array}{l}\text { Salvador } \\
\text { et al. } \\
(2011)\end{array}$ & $\begin{array}{l}\text { En-A } \\
\text { (RSPQ-2F). } \\
\text { ME (Escala } \\
\text { ad hoc) }\end{array}$ & $\begin{array}{l}\text { Se halló una tendencia armonizadora en los estudian- } \\
\text { tes entre las preferencias de aprendizaje y métodos de } \\
\text { enseńanza en toma de apuntes, pruebas objetivas y eva- } \\
\text { luación mediante trabajos-proyectos individuales. Se } \\
\text { hallaron tendencias discrepantes entre la preferencia por } \\
\text { las dinámicas grupales de los alumnos y el uso de los } \\
\text { docentes. Asimismo, esto se aplicó a las preferencias por } \\
\text { el libro de texto, los vídeos, la metodología del estudio } \\
\text { de casos, las preguntas cortas, los ejercicios o casos prác- } \\
\text { ticos y la modalidad virtual de enseñanza. }\end{array}$ \\
\hline $\begin{array}{l}\text { Villalustre } \\
\text { Martínez y } \\
\text { Del Moral } \\
\text { Pérez } \\
(2011)\end{array}$ & $\begin{array}{l}\text { Es-A (CHAEA). } \\
\text { SAD (Escala } \\
\text { ad hoc) }\end{array}$ & $\begin{array}{l}\text { Los estudiantes con estilos preferentes tipo pragmático y } \\
\text { activo manifestaron un mayor nivel de satisfacción con } \\
\text { la realización de mapas mentales. No obstante, aquellos } \\
\text { estudiantes con preferencias cognitivas tipo reflexivo } \\
\text { han subrayado el hecho de que las dinámicas plantea- } \\
\text { das en la materia han sido más satisfactorias que para el } \\
\text { resto del alumnado. }\end{array}$ \\
\hline
\end{tabular}

Nota: * Número de identificación de los artículos consultados.

En líneas generales, estos artículos comparten la visión de que es necesario conocer cómo se enfrentan los estudiantes al aprendizaje en función de sus propias estrategias y de las conductas docentes de sus profesores (metodologías, recursos, evaluación) dentro de un contexto determinado. Se trataría, en lo posible, de la integración del proceso de autorregulación de los alumnos y el de los profesores (Berbén, Pichardo \& De La Fuente, 2007; Martínez Geijo, 2009; Ramírez Díaz \& Chávez Lima, 2010; Villalobos-Alarcón et al., 2009). De este modo, se propone que la reducción de incompatibilidades entre estilos se puede lograr mediante el empleo de una amplia variedad métodos y estrategias instruccionales por parte del docente que se adapten a la pluralidad de preferencias cognitivas de aprendizaje de los estudiantes (García Ros, Pérez González \& Talaya González, 2008; Pulido Martos, De la Torre-Cruz, Luque Ramos \& Palomo Monereo, 2009; Ramírez 
Díaz \& Chávez Lima, 2010; Salvador et al., 2011; Villalustre Martínez \& Del Moral Pérez, 2011).

En este sentido, Hernández Ruiz (2004, p. 13) planificó posibles actividades y su secuencia para abordar la correspondencia: (a) determinar los estilos de aprendizaje del profesor y de los estudiantes; (b) alternar los estilos de enseńanza, de modo que se produzca una adaptación de estilo profesor-alumno y alumno-profesor a través de una gran gama de actividades; (c) organizar actividades en equipo. De vez en cuando, el profesor puede organizar los equipos de acuerdo con los estilos de aprendizaje comunes, también puede formar grupos con estilos de aprendizaje diferentes para aumentar la eficiencia y generar mayor flexibilidad de estilos y comportamientos; y (d) incluir enfoques y actividades para los diferentes estilos de aprendizaje en el plan de clase. Cabe recordar que el profesor debe actuar como facilitador, promoviendo el fortalecimiento y diversidad de alternativas de estilos de aprendizaje de los estudiantes, usando una gran variedad de métodos y materiales de enseñanza, y creando un ambiente caracterizado por la diversidad y la colaboración.

Ante lo expuesto, es posible inferir que estos estudios se alinean a la corriente de la "instrucción adaptativa" (Corno \& Snow, 1986; García García; 1997; Snow, 1980) entendida como la necesidad del profesor de reconocer las diferencias individuales del alumnado. La tarea docente consiste en adecuar su actuación a las características de los alumnos, o ayudando a los alumnos a ajustar sus expectativas y comportamientos a las particularidades de los docentes. En síntesis, los artículos Tipo I asumen que la orientación del emparejamiento debe dirigirse del docente a los estudiantes. El equilibrio en la interacción docente-alumnos estaría dado por la correspondencia entre los métodos de enseñanza, las actividades o los materiales elaborados por el docente y las preferencias de estilo de los estudiantes. De aquí que el modo de concretarlo estaría encaminado hacia la diversificación de las metodologías de enseñanza del docente. 


\section{Análisis bidireccional sobre la interacción estilos de aprendizaje y enseñanza}

Se hallaron 7 informes de investigación sobre la interacción estilos de aprendizaje y de enseńanza desde una perspectiva bidireccional (véase Tabla 4). Debido a que el interés recae sobre la relación, el análisis involucra tanto las preferencias de los estudiantes así como de sus docentes, estableciendo grados de correspondencia entre los mismos. El período temporal de las investigaciones de este grupo se encuentra entre 2005-2012, incrementando su volumen progresivamente en los últimos años. La mayor parte de ellas se radicaron en el contexto universitario latinoamericano, especialmente, en Venezuela y Colombia.

\section{Tabla 4}

Características de los artículos Tipo II según año de publicación

Tipo II - Perspectiva bidireccional

\begin{tabular}{|c|c|c|c|}
\hline \multirow{2}{*}{ Año } & \multirow{2}{*}{$\begin{array}{l}\text { Contexto } \\
\text { universitario* }\end{array}$} & \multicolumn{2}{|l|}{ Tamańo y tipo de muestra } \\
\hline & & Estudiantes & Docentes \\
\hline 2005 & Venezuela & $\begin{array}{l}50 \text { estudiantes de } \\
\text { diversas carreras }\end{array}$ & $\begin{array}{l}14 \text { docentes de diversas } \\
\text { titulaciones. }\end{array}$ \\
\hline 2007 & Venezuela & $\begin{array}{l}41 \text { estudiantes de } \\
\text { medicina }\end{array}$ & 60 profesores de medicina \\
\hline 2008 & España & $\begin{array}{l}753 \text { estudiantes de } \\
\text { diversas carreras }\end{array}$ & $\begin{array}{l}42 \text { docentes de diferentes } \\
\text { carreras }\end{array}$ \\
\hline 2008 & Argentina & $\begin{array}{l}47 \text { estudiantes de } \\
\text { informática }\end{array}$ & $\begin{array}{l}\text { Docentes de informática. No } \\
\text { se informó el tamaño muestra }\end{array}$ \\
\hline 2009 & Colombia & $\begin{array}{l}112 \text { estudiantes de } \\
\text { psicología }\end{array}$ & 23 profesores de psicología \\
\hline 2009 & Colombia & $\begin{array}{l}55 \text { estudiantes de } \\
\text { medicina }\end{array}$ & $\begin{array}{l}\text { Docentes de medicina. No se } \\
\text { informó el tamańo muestra }\end{array}$ \\
\hline 2012 & Chile & $\begin{array}{l}241 \text { alumnos de } \\
\text { educación diferencial y } \\
\text { pedagogía en inglés }\end{array}$ & $\begin{array}{l}35 \text { docentes de educación } \\
\text { diferencial y pedagogía en } \\
\text { inglés }\end{array}$ \\
\hline
\end{tabular}

Nota: ${ }^{*}$ Filiación del primer autor del artículo 
Tal como puede apreciarse en la Tabla 5 , se presentan los referentes de cada artículo junto con una breve descripción de las investigaciones bidireccionales o tipo II (variables, instrumentos y resultados principales). A continuación, se integraron los modos de entender y abordar la correspondencia entre estilos según esta clase de artículos.

\section{Tabla 5}

Revisión sobre las investigaciones Tipo II

\begin{tabular}{|c|c|c|}
\hline Autores & $\begin{array}{l}\text { Variables e } \\
\text { instrumentos }\end{array}$ & Resultados principales \\
\hline $\begin{array}{l}\text { Castro y } \\
\text { Guzmán } \\
(2005)\end{array}$ & $\begin{array}{l}\text { Es-A/Es-E } \\
\text { (Entrevista. } \\
\text { Observación) }\end{array}$ & $\begin{array}{l}\text { Los estudiantes no conocen ni la teoría ni la } \\
\text { práctica de los estilos de aprendizaje, no cursan } \\
\text { ninguna asignatura que tenga ese contenido. }\end{array}$ \\
\hline $\begin{array}{l}\text { Bravo } \\
\text { Hernández } \\
\text { y Alfonso } \\
\text { Romero } \\
(2007)\end{array}$ & $\begin{array}{l}\text { Es-A (CHAEA) } \\
\text { GDD (Guías ad hoc) }\end{array}$ & $\begin{array}{l}\text { La mayoría de los estudiantes tienen un } \\
\text { estilo de aprendizaje reflexivo, mientras que } \\
\text { la mayor parte de las guías didácticas elabo- } \\
\text { radas por los profesores tenían preferencia } \\
\text { por el estilo teórico. Las guías didácticas } \\
\text { como recurso del aprendizaje en la formación } \\
\text { de profesionales de la salud es un aconteci- } \\
\text { miento que por primera vez los profesores } \\
\text { están afrontando, además, no consideraban } \\
\text { habitualmente los estilos de aprendizaje. }\end{array}$ \\
\hline $\begin{array}{l}\text { Gargallo } \\
\text { López } \\
\text { (2008) }\end{array}$ & $\begin{array}{l}\text { En-A (Study Process } \\
\text { Questionnaire) } \\
\text { Es-D (CEMEDEPU) }\end{array}$ & $\begin{array}{l}\text { Los profesores que trabajaban con meto- } \\
\text { dologías centradas en el aprendizaje tuvie- } \\
\text { ron alumnos que desarrollaban estrategias } \\
\text { de aprendizaje de más calidad y utilizaban } \\
\text { enfoques más profundos de aprendizaje. Sin } \\
\text { embargo, los resultados eran mucho más cla- } \\
\text { ros cuando los profesores, además de tener } \\
\text { una concepción constructivista disponían de } \\
\text { más habilidades docentes. De modo que se } \\
\text { puede afirmar que hay un factor modulador, } \\
\text { que es la disposición de habilidades docentes. }\end{array}$ \\
\hline
\end{tabular}




\begin{tabular}{|c|c|c|}
\hline Autores & $\begin{array}{l}\text { Variables e } \\
\text { instrumentos }\end{array}$ & Resultados principales \\
\hline $\begin{array}{l}\text { Durán y } \\
\text { Costaguta } \\
(2008)\end{array}$ & $\begin{array}{l}\text { Es-A (ILS) } \\
\text { Es-E (Diseño } \\
\text { experimental de } \\
\text { ajuste) }\end{array}$ & $\begin{array}{l}\text { La adecuación de las estrategias típicas de } \\
\text { enseñanza al estilo de aprendizaje dominante } \\
\text { de los estudiantes impactó de manera positiva } \\
\text { en el rendimiento académico de los mismos. } \\
\text { Especialmente, mejoró la comprensión de } \\
\text { conceptos, técnicas y métodos de la disciplina } \\
\text { así como las habilidades para la resolución de } \\
\text { problemas usando herramientas metodológi- } \\
\text { cas y tecnologías de la disciplina. }\end{array}$ \\
\hline $\begin{array}{l}\text { Gravini } \\
\text { Donado et } \\
\text { al. (2009) }\end{array}$ & $\begin{array}{l}\text { Es-A (CHAEA) } \\
\text { Est-E (Cuestionario } \\
\text { ad hoc) }\end{array}$ & $\begin{array}{l}\text { Los estudiantes prefirieron el estilo de apren- } \\
\text { dizaje teórico, que comprende la lectura de } \\
\text { materiales complejos, el resumen de teorías e } \\
\text { hipótesis dadas por otras personas, sintetizar y } \\
\text { analizar la información. En cuanto a las estrate- } \\
\text { gias de enseńanza de las que se presume tienen } \\
\text { relación con el estilo teórico de aprendizaje } \\
\text { de los estudiantes, se encuentran: el resumen, } \\
\text { seminario, los mapas conceptuales y la rela- } \\
\text { toría. Los docentes afirman que "siempre" } \\
\text { utilizan el resumen y "a veces" el seminario y } \\
\text { "los mapas conceptuales", lo cual indicaría } \\
\text { cierta correspondencia entre ambos estilos. }\end{array}$ \\
\hline $\begin{array}{l}\text { Ayala- } \\
\text { Pimentel et } \\
\text { al. (2009) }\end{array}$ & $\begin{array}{l}\text { Es-A (CHAEA) } \\
\text { Es-E (Diseño } \\
\text { experimental de } \\
\text { ajuste) }\end{array}$ & $\begin{array}{l}\text { La mayor parte de los estudiantes prefirieron } \\
\text { el estilo de aprendizaje reflexivo. Obtuvieron } \\
\text { un mejor rendimiento académico aquellos } \\
\text { que conocían su estilo de aprendizaje y utili- } \\
\text { zaron como estrategias pedagógicas los mapas } \\
\text { conceptuales, los cuales permiten la orga- } \\
\text { nización jerárquica y visual de conceptos y } \\
\text { la constatación de sus relaciones con con- } \\
\text { ceptos previos. Su elaboración fomentó el } \\
\text { conocimiento, el pensamiento reflexivo, la } \\
\text { creatividad y el análisis crítico sobre un tema. } \\
\text { La utilización de situaciones-problema y la } \\
\text { formulación de secuencias neurofuncionales } \\
\text { consiguió un mejor aprendizaje de las estruc- } \\
\text { turas nerviosas y sus conexiones. }\end{array}$ \\
\hline
\end{tabular}




\begin{tabular}{|c|c|c|}
\hline Autores & $\begin{array}{l}\text { Variables e } \\
\text { instrumentos }\end{array}$ & Resultados principales \\
\hline $\begin{array}{l}\text { Catalán } \\
\text { Henríquez et } \\
\text { al. (2012) }\end{array}$ & $\begin{array}{l}\text { Es-A (CHAEA) } \\
\text { Est-E (Cuestionario } \\
\text { ad hoc) }\end{array}$ & $\begin{array}{l}\text { Los profesores y alumnos manifiestan una } \\
\text { tendencia preferentemente moderada por } \\
\text { el estilo teórico, es decir, suelen adaptar e } \\
\text { integrar las observaciones en teorías lógicas } \\
\text { y complejas. Prefieren analizar y sintetizar. } \\
\text { Buscan la racionalidad y objetividad en sus } \\
\text { acciones, decisiones y actividades. Los docen- } \\
\text { tes declaran que las estrategias de enseńanza } \\
\text { utilizadas preferentemente favorecen los cua- } \\
\text { tro estilos de aprendizaje, con un leve énfasis } \\
\text { por las que promueven el estilo Teórico. } \\
\text { Esto se condice con el estilo de aprendizaje } \\
\text { que más distingue a los alumnos de ambas } \\
\text { carreras. }\end{array}$ \\
\hline
\end{tabular}

Nota: ${ }^{*}$ Número de identificación de los artículos consultados

En estos artículos existe un consenso generalizado que los docentes enseñan de acuerdo a como les gustaría aprender y a las estrategias que les resultaron más eficaces para avanzar en la formación universitaria e introducirse en dicha comunidad disciplinar compartiendo formas de pensar y actuar. Desde esta perspectiva, se plantea no solo la importancia de identificar los estilos de los estudiantes sino también de considerar los estilos de aprendizaje de los docentes, en la medida en que estas preferencias impactarán sobre sus métodos de enseñanza habituales, incidiendo en consecuencia sobre las formas de aprender y el rendimiento estudiantil (Ayala-Pimentel, Díaz-Pérez \& OrozcoVargas, 2009; Catalán Henríquez, Lobos Salas \& Ortiz Aguilar, 2012; Gravini Donado et al., 2009). Explica Gargallo López (2008, p. 441): "Los alumnos utilizan determinados modos de aprender, más o menos estratégicos, más o menos profundos, que ajustan (no solo pero sí especialmente) en función de lo que hacen los profesores”. En este sentido, es posible enfatizar que, el alumno, conforme avanza en su proceso de aprendizaje se apropia de mejores formas en coherencia con la disciplina enseñada. 
Ante lo expuesto, es posible inferir que estos estudios se alinean a la corriente "estilística educativa" (Hederich Martínez, 2013) previamente denominada "enfoque estilístico" (Sternberg \& Grigorenko, 1995) que reconoce: (a) cada docente posee un estilo de aprendizaje, al igual que los estudiantes; (b) el estilo de aprendizaje del docente opera como un mediador en su estilo de enseñanza; (c) el estilo del profesor influye en las modalidades de aprendizaje de sus estudiantes, en las estructuras y dinámicas interactivas docente-estudiantes y en los resultados de aprendizaje.

En suma, los artículos Tipo II plantean que a orientación del emparejamiento debe dirigirse del docente a los estudiantes. Un primer emparejamiento necesario estaría dado por el ajuste entre las estrategias y estilos docentes y la naturaleza del contenido/tipo de conocimiento disciplinar. Un segundo emparejamiento sería el resultado de la adaptación cognitiva entre las mismas preferencias de los estudiantes y los estilos docentes predominantes. La forma de abordarlo sería establecer acuerdos entre los docentes acerca de los estilos de aprendizaje más adecuados para cada episteme y establecer programas de discusión, formación y orientación para docentes (Castro \& Guzman, 2005; Gravini Donado et al., 2009). Así como la inclusión de una disciplina de tipo transversal de corte instrumental en los primeros cursos de la universidad, que enseñe a los estudiantes a aprender a aprender en las que se pueden trabajar estrategias de aprendizaje (Gargallo López, 2008).

\section{Conclusiones}

La revisión efectuada de la literatura científica publicada en los últimos diez años en torno a la interacción entre estilos de aprendizaje y estilos de enseńanza en el ámbito universitario permite concluir que si bien su interés se ha difundido y diversificado, continúa en proceso de construcción. Esto se evidencia en los diferentes enfoques y modalidades de abordajes que proyectan encuentros y desencuentros entre los resultados y conclusiones que se derivan de sus análisis. Los puntos 
de mayor acuerdo radican en apoyar la hipótesis de ajuste instructivo basada en el supuesto de que la adquisición de conocimientos en el aula universitaria se encuentra mediada y facilitada por la correspondencia entre los estilos de aprendizaje predominantes del alumnado y el estilo de enseñanza de los docentes. Otro punto de acuerdo consiste en la necesidad de que el docente identifique las preferencias de aprendizaje de sus estudiantes para poder ayudar a crear un entorno efectivo para la transformación del aprendizaje. En contraposición, entre los puntos más álgidos del debate se sitúan dos formas de entender y abordar la correspondencia entre estilos: por un lado, la línea de la instrucción adaptativa que se expresa en la mayor parte de los artículos hallados y agrupados como unidireccionales que proponen el ajuste del estilo docente a las preferencias cognitivas de sus alumnos. Esto representa la correspondencia entre los métodos de enseñanza, las actividades o los materiales elaborados por el docente y los estilos predominantes de los estudiantes.

Por otro lado, se encuentra el enfoque estilístico que se manifiesta mediante los artículos agrupados como bidireccionales que asumen que el docente debería reconocer su propio estilo de aprendizaje, en la medida en que este estilo impactará sus métodos de enseñanza preferidos, incidiendo directamente sobre las formas habituales en que los estudiantes deberían aprender en ese contexto. Esta orientación se identificaría con el tipo de ajuste propuesto por Clariana (2001) basado en la correspondencia entre las mismas preferencias o estilos entre docentes y estudiantes. En suma, la revisión aquí realizada ha permitido explicitar que, a pesar de encontrar numerosos estudios en el tema de los estilos de aprendizaje y enseńanza en la universidad, poco se conoce sobre cómo se produce y/o debería generarse su ajuste.

Aguilar Rivera (2010) afirmó que "los estilos de aprendizaje son relativamente estables aunque pueden cambiar [...] la elección de un estilo depende también del perfil académico de la carrera elegida, pues hay carreras que demandan más un estilo que otro" (p. 224). En este sentido, a los fines de contribuir a la profundización de esta temática, se proponen como futuros estudios: analizar si las preferencias 
por los métodos instruccionales varían en función de la elección de la modalidad de estudios universitarios (García Ros et al., 2008) o actividades preprofesionales (Villalobos-Alarcón et al., 2009). De esta manera, se visualiza como un campo promisorio un mayor desarrollo investigativo para definir la relación entre estilos de aprendizaje y estrategias de enseñanza, ya que guardan altos grados de articulación al punto de que uno no se puede presentar sin el otro. El aprendizaje no se da en el vacío, sino a través de las estrategias y mediaciones contextuales de las que el docente se vale en su proceso de enseñanza (Gravini Donado et al., 2009).

\section{Referencias}

Aguilar Rivera, M. C. (2010). Estilos y estrategias de aprendizaje en jóvenes ingresantes a la universidad. Revista de Psicología, 28(2), 207-226.

Ayala-Pimentel, J. O., Díaz-Pérez, J.A. \& Orozco-Vargas, L. C. (2009). Eficacia de la utilización de estilos de aprendizaje en conjunto con mapas conceptuales y aprendizaje basado en la resolución de problemas para el aprendizaje de neuroanatomía. Educación Médica, 12(1), 25-31.

Bahamón Muñetón, M., Vianchá Pinzón, M., Alarcón Alarcón, L. \& Bohórquez Olaya, C. (2012). Estilos y estrategias de aprendizaje: Una revisión empírica y conceptual de los últimos diez años. Pensamiento Psicológico, 10(1), 129-144.

Berbén, A., Pichardo, M. \& De La Fuente, J. (2007). Relaciones entre preferencias de la enseńanza y enfoques de aprendizaje de los universitarios. Infancia y Aprendizaje, 30(4), 537-550.

Bertoglia Richards, L. (2005). La interacción profesor-alumno. Una visión desde los procesos atribucionales. Psicoperspectivas. Individuo y Sociedad, 4, 57-73. 
Blumen, S., Rivero, C. \& Guerrero, D. (2011). Universitarios en educación a distancia: estilos de aprendizaje y rendimiento académico. Revista de Psicología, 29(2), 225-243.

Bravo Hernández, P. \& Alfonso Romero, M. (2007). Comportamiento de actividades que desarrollan estilos de aprendizaje en las guías didácticas de la asignatura Morfo-fisiología Humana I. Educación Médica Superior, 21(4), 1-10.

Castro, S. \& Guzmán, B. (2005). Los estilos de aprendizaje en la enseñanza y el aprendizaje: Una propuesta para su implementación. Revista de investigación, 58, 83-102.

Catalán Henríquez, S., Lobos Salas, C. \& Ortiz Aguilar, V. (2012). Diagnóstico de los estilos de aprendizaje de los alumnos y profesores de las carreras de Pedagogía en Inglés y Educación Diferencial, UST Viña del Mar. Revista Estilos de Aprendizaje, 9, 1-17.

Clariana, M. (2001). Las preferencias instruccionales: Conceptualización y evaluación. Revista de Psicología General y Aplicada, 54(2), 259-277.

Corno, L. \& Snow, R. (1986). Adapting teaching to individual differences among learners. En M. Wittrock (Ed.), Handbook of research on teaching (pp. 605-629). Nueva York, USA: McMillan.

Durán, E. \& Costaguta, R. (2008). Experiencia de enseñanza adaptada al estilo de aprendizaje de los estudiantes en un curso de simulación. Formación Universitaria, 1(1), 19-28.

Esguerra Pérez, G. \& Guerrero Ospina, P. (2010). Estilos de aprendizaje y rendimiento académico en estudiantes de Psicología. Diversitas. Perspectivas en psicología, 6(1), 97-109.

Evans, C., Cools, E. \& Charlesworth, Z. M. (2010). Learning in higher education: How cognitive and learning styles matter. Teaching in Higher Education, 15(4), 467-478.

Gargallo López, B. (2008). Estilos de docencia y evaluación de los profesores universitarios y su influencia sobre los modos de aprender de sus estudiantes. Revista Española de Pedagogía, 241, 425-446. 
García García, M. (1997). Educación adaptativa. Revista de Investigación Educativa, 15(2), 247-271.

García Ros, R., Pérez González, F. \& Talaya González, I. (2008). Preferencias respecto a métodos instruccionales de los estudiantes universitarios de nuevo acceso y su relación con estilos de aprendizaje y estrategias motivacionales. Electronic Journal of Research in Educational Psychology, 6(16), 547-570.

González Lomelí, D., Castañeda Figueiras, S. \& Maytorena Noriega, M. A. (2000). Estilos de aprendizaje y aprovechamiento en ingresantes universitarios. Revista de Psicología, 18(2), 199-225.

Gravini Donado, M., Cabrera Pérez, E., Ávila Molina, V. \& Vargás González, I. (2009). Estrategias de enseñanza en docentes y estilos de aprendizaje en estudiantes del programa de psicología de la Universidad Simón Bolívar, Barranquilla. Revista Estilos de Aprendizaje, 3, 124-140.

Hederich Martínez, C. (2013). Estilística educativa. Revista Colombiana de Educación, 64, 21-56.

Hederich Martínez, C., Gravini Donado, M. \& Camargo Uribe, A. (2011). El estilo y la enseñanza: Un debate sobre cómo enfrentar las diferencias individuales en el aula de clase. En R. Roig Vila y C. Laneve, C. (Eds.), La pratica educativa nella società dellinformazione. Linnovazione attraverso la ricerca (pp. 213222). Alcoy-Brescia, Italia: Marfil y La Scuola Editrice.

Hernández Ruiz, L. (2004). La importancia de los estilos de aprendizaje en la enseńanza de inglés como lengua extranjera. Espéculo: Revista de Estudios Literarios, 27, 1-15.

Hervás Aviles, R. (2003). Estilos de enseñanza y aprendizaje en escenarios educativos. Granada, España: Grupo Editorial Universitario.

Joyce, B., Weil, M. \& Calhoun, E. (2000). Models of teaching. Washington, USA: Pearson.

Komarraju, M., Karau, S. J., Schmeck, R. R. \& Avdic, A. (2011). The Big Five personality traits, learning styles, and academic achievement. Personality and Individual Differences, 51, 472477. 
Martínez Geijo, P. (2008). Aprender y enseñar los estilos de aprendizaje y de enseñanza desde la práctica de aula. Bilbao, España: Mensajero.

Martínez Geijo, P. (2009). Estilos de enseñanza: Conceptualización e investigación (en función de los estilos de aprendizaje de Alonso, Gallego y Honey). Revista Estilos de Aprendizaje, 3, 3-19.

Peterson, E. R., Rayner, S. G. \& Armstrong, S. J. (2009). Researching the psychology of cognitive style and learning style: Is there really a future? Learning and Individual Differences, 19, 518-523.

Prados Gallardo, M., Cubero Pérez, M. \& de la Mata Benítez, M. (2010). ¿Mediante qué estructuras interactivas se relacionan profesorado y alumnado en las aulas universitarias? Electronic Journal of Research in Educational Psychology, 8(1), 163-194.

Pulido Martos, M., De la Torre-Cruz, M., Luque Ramos, P. \& Palomo Monereo, A. (2009). Estilos de enseñanza y aprendizaje en el EEES: Un enfoque cualitativo. Revista Estilos de Aprendizaje, 4, 156-171.

Ramírez Díaz, M. \& Chávez Lima, E. (2010). Análisis de la influencia del estilo de enseñanza del profesor en el aprendizaje de estudiantes de física a nivel universitario. Latin-American Journal of Physics Education, 4(Supl.1), 1002-1008.

Ruiz Ruiz, B., Trillos Gamboa, J. \& Morales Arrieta, J. (2006). Estilos de aprendizaje y rendimiento académico en estudiantes universitarios. Revista Galego-Portuguesa de Psicoloxía e Educación, 11-12(13), 441-457.

Salvador, L., Argos González, J., Ezquerra Muñoz, M., Osoro Sierra, M. \& Castro, A. (2011). Perfiles de estilos de aprendizaje de los estudiantes universitarios y metodologías docentes. Bordón. Revista de Pedagogía, 63(2), 41-52.

Serrano Pastor, F. (1994). La interacción estilos de aprendizaje-estilos de enseñanza: Emparejamiento versus no-emparejamiento. Anales de Pedagogía, 12-13, 81-112.

Snow, R. E. (1980). Aptitude, learner control, and adaptive instruction. Educational Psychologist, 15(3), 151-158. 
Sternberg, R. \& Grigorenko, E. (1995). Styles of thinking in the school. European Journal of High Ability, 6(2), 201-219.

Villalobos-Alarcón, E., Guerrero-Faquíez, M., Pérez-Villegas, R., Avendaño-Veloso, A., Ceballos-Morales, A., Ortiz-Contreras, J. \& Parra-Ponce, C. (2009). Estilos de aprendizaje y metodologías de enseñanza en estudiantes de obstetricia. Educación Médica, 12(1), 43-46.

Villalustre Martínez, L. \& Del Moral Pérez, M. (2011). E-actividades en el contexto virtual de rural-net: Satisfacción de los estudiantes con diferentes estilos de aprendizaje. Educación XX1, 14(1), 223-243.

Recibido: 16 de agosto, 2013

Aceptado: 27 de agosto, 2013 